\title{
Commentary: Management of Refractory Pain After Total Joint Replacement
}

\author{
Jamie Heimroth' ${ }^{*}$, Max L. Willinger', Nipun Sodhi' ', Luke J. Garbarino', Peter A. Gold', Jonathan R. Danoff', \\ Sreevathsa Boraiah ${ }^{2}$ \\ 'Department of Orthopedic Surgery, Long Island Jewish Medical Center, Queens, NY, USA \\ 2Department of Orthopedic Surgery, North Shore University Hospital, Manhasset, NY, USA
}

\section{Article Info}

\section{Article Notes}

Received: June 30, 2021

Accepted: September 08, 2021

\section{${ }^{*}$ Correspondence:}

${ }^{*}$ Dr. Jamie Heimroth, Department of Orthopedic Surgery, Long Island Jewish Medical Center, Queens, NY, USA; Email: jheimroth1@northwell.edu

${ }^{\oplus} 2021$ Heimroth J. This article is distributed under the terms of the Creative Commons Attribution 4.0 International License.

\section{Keywords:}

Refractory pain

Chronic pain

Total joint arthroplasty

Knee osteoarthritis

Postoperative analgesia

Genicular nerve radiofrequency ablation

\section{Abstract}

Chronic refractory pain after total joint replacement is debilitating and a source of dissatisfaction for patients. The management of pain following total joint replacement varies during the acute postoperative period compared to the 3 months postoperative at which point the pain is considered chronic pain. Acute postoperative pain relief programs have seen promising results with multimodal pain control through the use of combinations of opioids, acetaminophen, nonsteroidal anti-inflammatory drugs, gabapentinoids and ketamine. The addition of regional blocks to the multimodal regimen has improved acute postoperative pain control following total joint replacements. On the other hand, chronic pain can be successfully managed with options including genicular nerve radioablation therapy (GN-RFA), neuromuscular electrical stimulation (NMES), transcutaneous electrical nerve stimulation (TENS), and peripheral subcutaneous field stimulation (PSFS). While there is still minimal data on chronic pain relief regimens, meta-analyses and case reports have demonstrated the effectiveness and promising outcomes. This paper aims to evaluate the current medications and treatment options for managing refractory pain following TJA.

\section{Introduction}

Total joints replacements (TJA) are proven treatment options for severe osteoarthritis. While total joint replacements have high satisfaction rates of up to $80 \%$, there are still $20 \%$ of patients that are dissatisfied. Both acute and chronic pain following total joint replacements have the greatest impact on patient satisfaction. Previous studies have shown that up to $15 \%$ of patients that undergo total knee arthroplasty have residual moderate to severe pain that can persist for up to 5 years after the knee replacement ${ }^{1}$. Wylde et al. has found that $6 \%$ of patients undergoing total hip replacements had persistent severe pain ${ }^{1}$. The management of refractory postoperative pain is an essential tool, as medicine moves towards patient centered care and improving patient satisfaction following surgery. Willinger et al. conducted a comprehensive review of current literature on treatment options for managing refractory pain after TJA, this paper aims to highlight the conclusions of that literature review ${ }^{2}$.

\section{Acute Postoperative Pain Control}

Perioperative pain management is critical to patient care since addressing early postoperative pain can lead to decreased occurrence of refractory chronic pain syndrome especially in patients who are not opioid-naïve ${ }^{3,4,5}$. The objective of acute postoperative pain relief is to allow for early ambulation, improved patient satisfaction 
and decreased hospital lengths of stay. Pain relief can be provided through the use of medications such as opioids, acetaminophen, nonsteroidal anti-inflammatory drugs, gabapentinoids and ketamine.

The goal of postoperative pain management is to provide analgesia while minimizing the potential side effects of administering the medications. Each pain medication has a different side effect such as opioids' effect on respiratory depression and sedation ${ }^{2}$. Despite their side effects, opioids are effective analgesics that are often given on a postoperative dosing schedule $e^{6,7}$. The problem with opioid postoperative pain control coverage is that less than half of patients undergoing surgical procedures report adequate postoperative pain relief ${ }^{8}$. The reason for this poor pain control with prescribing only opioid medications postoperatively is that providers prescribe lower doses due to concerns over side effects leading to suboptimal dosing levels ${ }^{6}$. The poor postoperative pain relief then leads to worsening patient satisfaction and increased hospital lengths of stay and decreased early ambulation ${ }^{6}$. Providers need to administer adequate opioid dosage or administer multimodal pain medications to acquire appropriate pain relief.

Multimodal pain control aims to provide analgesia through a combination of pain medications. The goal is to administer a combination of medications that work via different pathways such as NSAIDS that inhibit the production of prostaglandins in the peripheral tissues reducing inflammation and opioids that act on mu, kappa and lambda receptors attenuate pain related signals to the central nervous system. Thybo et al. showed that combinations of medications such as ibuprofen and paracetamol led to a reduction in opioids in the acute postoperative period ${ }^{9}$. While randomized controlled trials have shown that multimodal pain control is superior to opioids alone, there is still debate over which medications should be used in the multimodal pain regimen ${ }^{10}$. Lamplot et al. added periarticular regional blocks to their multimodal pain regimen resulting in lower VAS scores, fewer adverse effects and lower narcotic use when compared to the patients receiving hydromorphone patient-controlled analgesia ${ }^{10}$. There are different options for regional nerve blocks for total knee arthroplasty that include femoral nerve block, adductor canal block and periarticular injection. A randomized control trial by Kim et al. showed that adductor canal blocks are not inferior to femoral nerve blocks for providing analgesia and decreasing opioid intake ${ }^{11}$. Kim et al. showed that patients had higher patient satisfaction and less opioid consumption when periarticular nerve blocks were augmented with infiltration between the popliteal artery and capsule of the posterior knee and adductor canal blocks ${ }^{12}$. The addition of regional blocks to the perioperative multimodal pain approach decreases acute postoperative pain leading to a decrease in refractory pain.

\section{Chronic Refractory Pain Control}

Chronic pain occurs when postsurgical pain lasts for longer than 3 months following surgery ${ }^{13}$. Treatment options for chronic pain management after total joint arthroplasty include genicular nerve radiofrequency ablation, transcutaneous electrical nerve stimulation (TENS), peripheral subcutaneous field stimulation (PSFS), and neuromuscular electrical stimulation (NMES). Genicular nerve radiofrequency ablation was originally used to treat chronic osteoarthritic knee pain in patients who were poor surgical candidates and has now shifted as a treatment option for chronic pain following total knee arthroplasty. Some of the problems that can occur with genicular nerve radiofrequency ablations are skin burns, and iatrogenic vascular injuries ${ }^{14}$. Of the nerve stimulation options, transcutanteous electrical nerve stimulation is the most studied following TJA resulting in improved Visual Analog Scale and Knee Injury and Osteoarthritis Outcome scores ${ }^{2}$. TENS have few side effects such as skin burns, and have no potential for overdose so patients have the opportunity to titrate the treatment ${ }^{15}$. Similar to TENS, peripheral subcutaneous field stimulation has also shown promising results when treating chronic pain after TJA. PSFS was previously used to treat neuropathic pain such as phantom limb pain. PSFS leads can be left indwelling for extended time periods without infection ${ }^{16}$. Lead fragmentation is a risk with PSFS, however, Ilfeld et al. showed no increase in infectious or neurologic complications from lead fragmentation ${ }^{16}$. As with TENS and PSFS, NMES use electrodes to stimulate a target such as the quadriceps muscles. Studies have shown improved pain and function scores when NMES are used to treat knee osteoarthritis. Delanois et al. showed that NMES can be used to improve pain scores, improve quadriceps lag, and improve timed up and go in acute postoperative patients, however, further studies need to be conducted to assess NMES use for treating refractory pain ${ }^{17}$.

\section{Conclusion}

Chronic pain following total joint arthroplasty is experienced by 8 to $34 \%$ of patients. Options for treating both chronic pain and acute pain after total joint arthroplasty are highlighted in Figure 1. The use of multimodal analgesia through combinations of acetaminophen, NSAIDs, opioids, ketamine, gabapentinoids and regional blocks has proven to be effective at managing both acute postoperative pain and chronic refractory pain. Multimodal analgesia regimens vary from institution to institution with different dosages of medications, timing of medications, and type of regional block administered. 


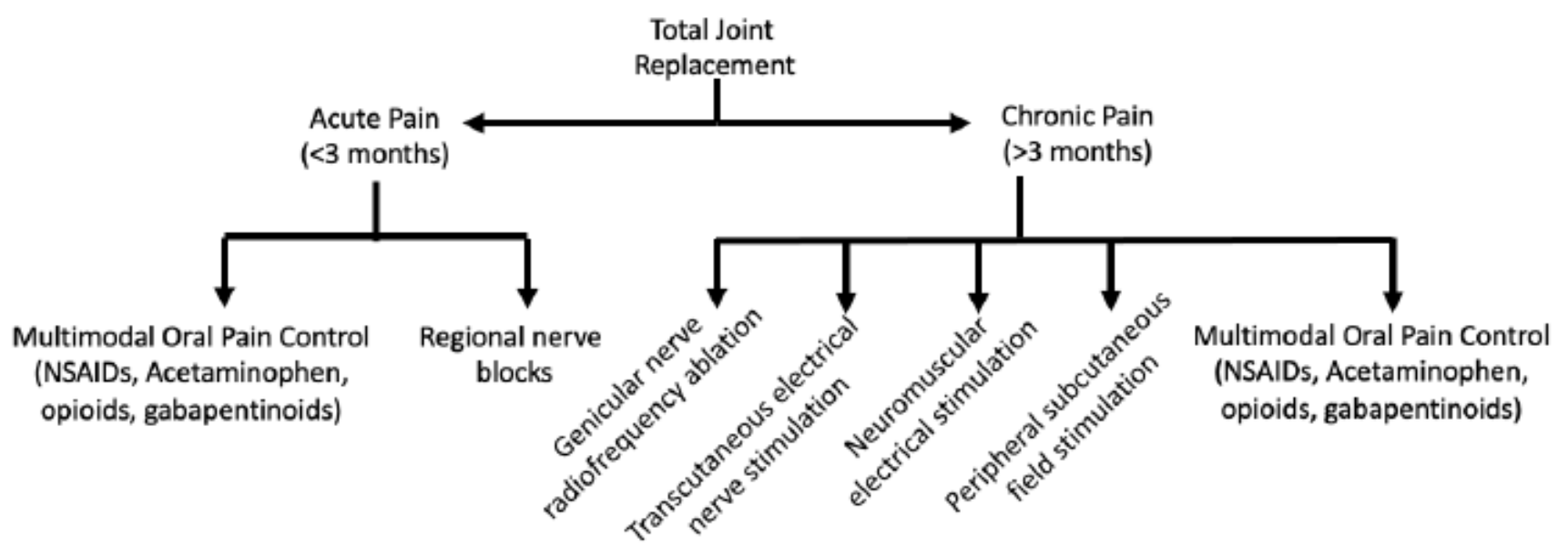

Figure 1: Postoperative pain management options following total joint replacements

While acute postoperative management continues to improve, focus has shifted towards identifying preoperative factors leading to increased postoperative pain. Kim et al. showed that preoperative pain and preoperative function are associated with the highest occurrence of chronic refractory postoperative pain following TJA which has also been seen in other surgeries such as hysterectomy, prostatectomy and lower extremity amputations ${ }^{13}$. Improving preoperative function through the use of preoperative physiotherapy with continued postoperative physiotherapy sessions leads to improved hip strength, range of movement and walking distance following total hip replacements ${ }^{18}$. Understanding that preoperative factors contribute to increased postoperative pain, there has been a shift towards focusing on identifying predictors of postoperative pain. Some predictors include preoperative pain, anxiety, depression, young age, obesity and type of surgery ${ }^{1,19}$. Pan et al. showed that patients undergoing total knee arthroplasty with anxiety, depression or both had increased postoperative pain compared to patients without anxiety or depression ${ }^{20}$. Treatment of preoperative conditions such as anxiety, depression and preoperative pain can decrease the likelihood of the patient having chronic refractory pain.

Patients that have chronic refractory pain following TJA have shown improvement with genicular nerve radiofrequency ablation, neuromuscular electrical stimulation, transcutaneous electrical nerve stimulation and peripheral subcutaneous field stimulation. The literature is limited on chronic pain treatment using these methods. Previous studies lack power secondary to small sample sizes and there are no randomized control trials comparing the chronic pain treatment options. One of the reasons for the lack of research is due to the difficulty of identifying patients suffering from chronic pain which can present in nontraditional ways. Further research into the development of chronic postoperative pain control tools and pain regimens is a priority for patients and healthcare systems as the number of patients with chronic refractory pain increases as total joint replacements continue to rise each year.

\section{Conflicts of Interest}

None of the authors have any conflicts of interest with the research.

\section{References}

1. Wylde V, Hewlett S, Learmonth ID, et al. Persistent pain after joint replacement: prevalence, sensory qualities, and postoperative determinants. PAIN. 2011; 152(3): 566-72.

2. Willinger ML, Heimroth J, Sodhi N, et al. Management of Refractory Pain After Total Joint Replacement. Current Pain and Headache Reports. 2021; 25(6): 1-9.

3. Jackman C. Perioperative pain management for the chronic pain patient with long-term opioid use. Orthopaedic Nursing. 2019; 38(2): 159-63.

4. Kehlet H, Jensen TS, Woolf CJ. Persistent postsurgical pain: risk factors and prevention. The lancet. 2006; 367(9522): 1618-25.

5. Rajpal S, Gordon DB, Pellino TA, et al. Comparison of perioperative oral multimodal analgesia versus IV PCA for spine surgery. Clinical Spine Surgery. 2010; 23(2): 139-45.

6. Parvizi J, Miller AG, Gandhi K. Multimodal pain management after total joint arthroplasty. JBJS. 2011; 93(11): 1075-84.

7. Horlocker TT. Pain management in total joint arthroplasty: a historical review. Orthopedics. 2010; 33(9): 14-9.

8. Chou R, Gordon DB, de Leon-Casasola OA, et al. Management of Postoperative Pain: a clinical practice guideline from the American pain society, the American Society of Regional Anesthesia and Pain Medicine, and the American Society of Anesthesiologists' committee on regional anesthesia, executive committee, and administrative council. The journal of pain. 2016; 17(2): 131-57.

9. Thybo KH, Hägi-Pedersen D, Dahl JB, et al. Effect of combination of paracetamol (acetaminophen) and ibuprofen vs either alone on patient-controlled morphine consumption in the first 24 hours after total hip arthroplasty: the PANSAID randomized clinical trial. Jama. 2019; 321(6): 562-71.

10. Lamplot JD, Wagner ER, Manning DW. Multimodal pain management in total knee arthroplasty: a prospective randomized controlled trial. The Journal of arthroplasty. 2014; 29(2): 329-34. 
11. Kim DH, Lin Y, Goytizolo EA, et al. Adductor canal block versus femora nerve block for total knee arthroplasty: a prospective, randomized, controlled trial. Anesthesiology. 2014; 120(3): 540-50.

12. Kim DH, Beathe JC, Lin Y, et al. Addition of infiltration between the popliteal artery and the capsule of the posterior knee and adductor canal block to periarticular injection enhances postoperative pain control in total knee arthroplasty: a randomized controlled trial. Anesthesia \& Analgesia. 2019; 129(2): 526-35.

13. Kim DH, Pearson-Chauhan KM, McCarthy RJ, et al. Predictive factors for developing chronic pain after total knee arthroplasty. The Journal of arthroplasty. 2018; 33(11): 3372-8.

14. Kidd VD, Strum SR, Strum DS, et al. Genicular nerve radiofrequency ablation for painful knee arthritis: the why and the how. JBJS essential surgical techniques. 2019; 9(1): e10.

15. Johnson M. Transcutaneous electrical nerve stimulation: mechanisms, clinical application and evidence. Reviews in pain. 2007; 1(1): 7-11.
16. Ilfeld BM, Ball ST, Gabriel RA, et al. A feasibility study of percutaneous peripheral nerve stimulation for the treatment of postoperative pain following total knee arthroplasty. Neuromodulation: Technology at the Neural Interface. 2019; 22(5): 653-60.

17. Delanois R, Sodhi N, Acuna A, et al. Use of home neuromuscular electrical stimulation in the first 6 weeks improves function and reduces pain after primary total knee arthroplasty: a matched comparison. Annals of translational medicine. 2019; 7(Suppl 7): S254.

18. Ackerman IN, Bennell KL. Does pre-operative physiotherapy improve outcomes from lower limb joint replacement surgery? A systematic review. Australian Journal of Physiotherapy. 2004; 50(1): 25-30.

19. $\mathrm{Wu} C L$, Raja SN. Treatment of acute postoperative pain. The lancet 2011; 377(9784): 2215-25.

20. Pan X, Wang J, Lin Z, et al. Depression and anxiety are risk factors for postoperative pain-related symptoms and complications in patients undergoing primary total knee arthroplasty in the United States. The Journal of arthroplasty. 2019; 34(10): 2337-46. 